\title{
Fetal transesophageal echocardiography during balloon valvuloplasty for severe aortic valve stenosis at $28+6$ weeks of gestation
}

Thomas Kohl, MD, ${ }^{\mathrm{a}, \mathrm{b}}$ Johannes Breuer, MD, ${ }^{\mathrm{c}}$ Axel Heep, MD, ${ }^{\mathrm{d}}$ Ingobert Wenningmann, MD, Julia Weinbach, MD, ${ }^{a}$ and Ulrich Gembruch, MD, ${ }^{a}$ Bonn and Münster, Germany

\section{Clinical Summary}

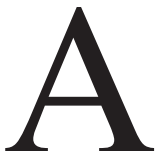

26-year old pregnant women had been referred to our center at $28+1$ weeks of gestation. Maternal transabdominal fetal ultrasound imaging at that time revealed severe fetal aortic valve stenosis with a poorly contracting, dilated left ventricle, moderate-to-severe mitral valve regurgitation, and local hyperechogenicity of the endocardium. Left-to-right shunting across the oval foramen and retrograde flow inside the aortic arch heralded loss of left ventricular function with ongoing pregnancy. ${ }^{1}$

From the German Center for Fetal Surgery \& Minimally-Invasive Therapy ${ }^{\mathrm{a}}$ and the Departments of Pediatric Cardiology, ${ }^{\mathrm{c}}$ Neonatal Intensive Care, ${ }^{\mathrm{d}}$ and Anesthesiology, ${ }^{\mathrm{e}}$ University Hospital of Bonn, Bonn, Germany; and the Münster University Children's Hospital, Department of Cardiology, ${ }^{\mathrm{b}}$ Münster, Germany

The development of percutaneous fetoscopic techniques for fetal cardiac interventions has been supported by educational and research grants (Ko 1484/1-1, Ko 1484/2-1, Ko 1484/3-1, Ko 1484/3-2, Ko 1484/3-3) of the Deutsche Forschungsgemeinschaft (DFG), Bonn, Germany. Dr Kohl reports consulting and lecture fees from Storz and Acuson-Siemens.

Received for publication Jan 6, 2006; revisions received Jan 19, 2006; accepted for publication Jan 29, 2006.

Address for reprints: Thomas Kohl, MD, German Center for Fetal Surgery \& Minimally-Invasive Therapy, Department of Obstetrics \& Prenatal Medicine, University of Bonn Medical School, 53105 Bonn, Germany (E-mail: thomas.kohl@ukb.uni-bonn.de)

J Thorac Cardiovasc Surg 2007;134:256-7

$0022-5223 / \$ 32.00$

Copyright $\odot 2007$ by The American Association for Thoracic Surgery doi:10.1016/j.jtcvs.2007.01.086
Percutaneous ultrasound-guided fetal balloon valvuloplasty was performed at $28+6$ weeks of gestation to salvage fetal left ventricular function. The procedure was viewed as an experimental treatment approach with the potential to improve quality of life and overall prognosis. Fetal transesophageal echocardiography (TEE) with a multimodal intravascular ultrasound catheter was used to improve imaging of device manipulation inside the fetal heart and aorta. $^{2}$ The ultrasound and fetoscopy-guided procedure was performed after parental informed consent and approval by the local committee of human research and in accordance with the ethical standards for human experimentation established by the Declaration of Helsinki.

The procedure was performed under general maternofetal anesthesia with desflurane and remifentanil. An 11F catheter sheath was percutaneously placed into the amniotic cavity above the fetal head and was steered into the fetal esophagus by fetoscopy. After these manipulations, a 10F single-plane intravascular ultrasound catheter (AcuNav; Acuson-A-Siemens Company, Erlangen, Germany) was inserted via the catheter sheath into the fetal esophagus and placed behind the fetal heart. The catheter is tipped with a frequency agile 5.5- to $10-\mathrm{MHz}$ vector phased-array ultrasound transducer that permits high-resolution 2-dimensional real-time imaging as well as multimodal Doppler imaging in a single plane that extends parallel to the long axis of the catheter up to $10 \mathrm{~cm}$ from the lens in the lateral direction. After the setup for
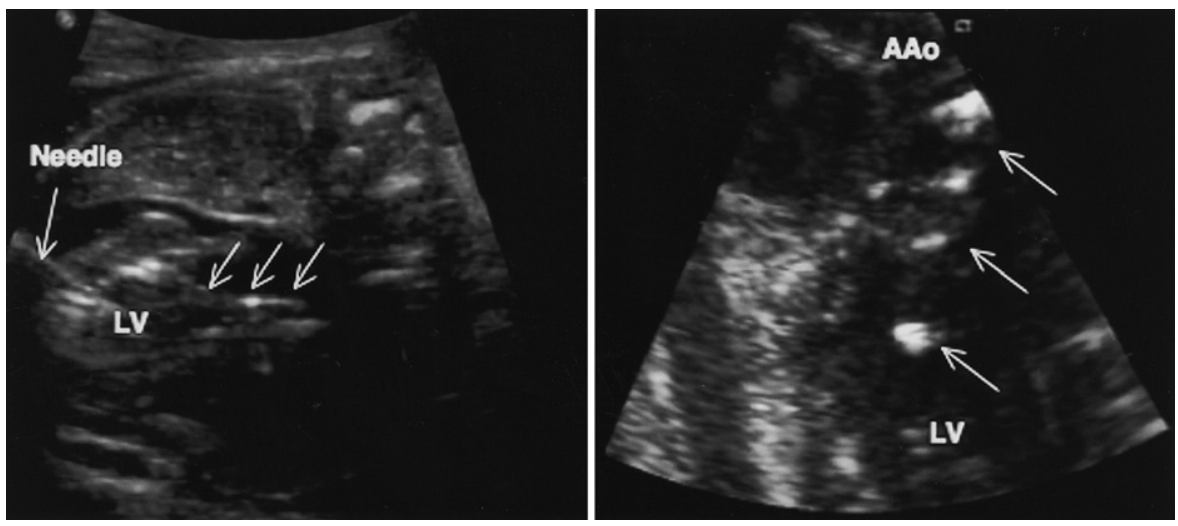

Figure 1. Conventional maternal transabdominal fetal echocardiography image (left) and fetal TEE image (right) during inflation of the balloon (arrows in both images point at balloon position across the aortic valve). Note that the conventional maternal transabdominal ultrasound images demonstrate significantly less detail than the fetal TEE ones. $L V$, Left ventricle; $A A o$, ascending aorta. 

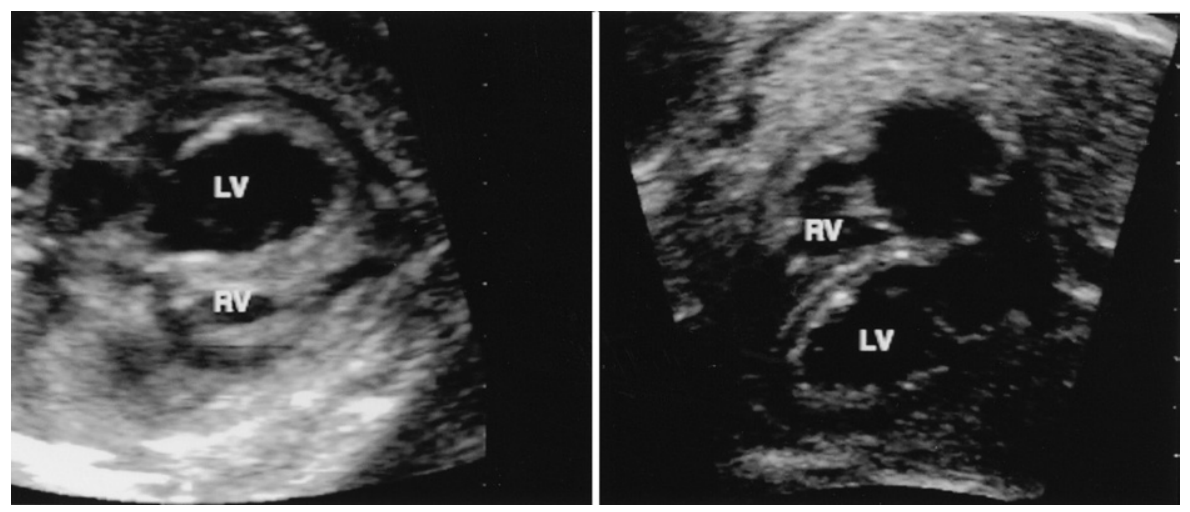

Figure 2. Left, Fetal 4-chamber view before intervention. Note the marked distention and hypertrophy of the left ventricle as well as the local hyperechogenicity of the endocardium. Right, Fetal 4-chamber view 3 days after the intervention. Note the remarkable normalization of the fetal cardiac aspect within this very short time. $L V$, Left ventricle; $R V$, right ventricle.

fetal TEE had been established, a 16-gauge needle was percutaneously inserted into the fetal heart and placed underneath the obstructed aortic valve. Successful balloon valvuloplasty was then achieved via the needle shaft with a coronary angioplasty catheter under combined maternal transabdominal and fetal TEE guidance (Figure 1). Mother and fetus tolerated the procedure well and complications were not observed. Within days after the procedure marked improvement of left ventricular function could be documented (Figure 2). The baby was delivered at 33 weeks of gestation and because of restenosis underwent redilatation of the aortic valve. In the meantime, the baby has been discharged home with a normal 2-ventricle circulation.

\section{Discussion}

Fetal TEE permitted substantially clearer definition of fetal cardiac anatomy and intracardiac device manipulation than had conventional maternal transabdominal fetal echocardiography. Because of the intrathoracic ultrasound catheter position right next to the fetal heart, sound attenuation by the maternal abdominal and uterine walls, amniotic fluid, as well as fetal chest bones become a nonissue during the novel imaging approach. High-quality 2dimensional imaging of all cardiac chambers, semilunar and atrioventricular valves, as well as supracardiac and infracardiac vessels can be achieved in vertical planes. Color and pulsed Doppler imaging permitted assessment of fetal cardiovascular flows and of the fetoplacental circulation. Depending on the orientation of the fetal cardiac apex, it may prove difficult to achieve low incidence angles for pulsed Doppler and color Doppler assessment of atrioventricular valve flows. In contrast, low incidence angles can be achieved for semilunar valves, great vessels, and assessment of the fetoplacental circulation.

During conventional maternal transabdominal fetal echocardiography, ultrasound scattering from the intracardiac needle shaft may overlay the stenotic valve and interfere with adequate visu- alization of guidewire and balloon catheter positioning (Figure 1). This obstacle can be entirely avoided by fetal TEE, during which scatter from interventional devices is always directed anteriorly and can never become situated between the obstructed semilunar valve and the posterior intraesophageal ultrasound transducer. As the fetal esophagus provides a rail for the ultrasound catheter, stable imaging planes that can reliably be reproduced are achieved by simple manipulations of the catheter (advance-withdrawal, left rotation-right rotation). Because the imaging planes during fetal TEE are vertically oriented, guidance of interventional devices across an obstructed fetal aortic valve is easily performed with great precision.

In addition to improving fetal cardiac imaging during percutaneous ultrasound-guided fetal balloon valvuloplasty, fetal TEE, in our opinion, will become a prerequisite during more complex minimally invasive fetal cardiac procedures such as fetoscopic pacemaker insertion that require gas insufflation of the amniotic cavity. ${ }^{3,4}$

\section{References}

1. Mäkikallio K, McElhinney DB, Levine JC, Marx GR, Colan SD, Marshall AC, et al. Fetal aortic valve stenosis and the evolution of hypoplastic left heart syndrome. Circulation. 2006;113:1401-5.

2. Kohl T, Westphal M, Strümper D, Achenbach S, Halimeh S, Petry P, et al. Multimodal fetal transesophageal echocardiography for fetal cardiac intervention in sheep. Circulation. 2001;114:1757-60.

3. Kohl T, Hering R, Van de Vondel P, Tchatcheva K, Berg C, Bartmann $\mathrm{P}$, et al. Analysis of the step-wise clinical introduction of experimental percutaneous fetoscopic surgical techniques for upcoming minimallyinvasive fetal cardiac interventions. Surg Endosc. 2006;20:1134-43.

4. Kohl T, Reckers J, Strümper D, Große Hartlage M, Gogarten W, Gembruch U, et al. Amniotic air insufflation during minimally invasive fetoscopic fetal cardiac interventions is safe for the fetal brain in sheep. J Thorac Cardiovasc Surg. 2004;128:467-71. 